\title{
DOUBLE-RESONANT SECOND HARMONIC GENERATION FROM SURFACE COVERAGES OF NILE-BLUE A
}

\author{
Gerd MAROWSKY, Alfred GIERULSKI and Bernhard DICK \\ Max-Planck-Institut für biophysikalische Chemie, Abteilung Laserphysik, \\ D-3400 Göttingen, Fed. Rep. Germany
}

Received 19 October 1984

Second harmonic emission from a dye covered quartz surface has been observed in total internal reflection. Coverages with the dye nile-blue $A$ yielded an increase in second harmonic output by several orders of magnitude as compared to coverages with rhodamine $6 \mathrm{G}$. In the latter case only the harmonic light is at resonance with the $S_{0}-S_{2}$ transition of the adsorbed dye molecules, whereas for nile-blue $\mathrm{A}$ both, the fundamental and the harmonic frequency, are at resonance with the respective singlet transitions.

\section{Introduction}

Since the pioneering work of Shen and coworkers [1-5] on nonlinear optical phenomena at solid and liquid interfaces, optically observable surface effects have gained increasing interest. In particular second harmonic (SH) generation has been developed to a versatile probe for surface analysis with respect to coverage density and molecular orientation $[6,7]$.

Second harmonic generation, which cannot occur in the bulk of centrosymmetric media for symmetry reasons within the electric dipole approximation [8], is restricted to the symmetry-breaking surface layer. Its intensity can be considerably increased by constructive interferometric enhancement $[9,10]$ or by adsorption of dye monolayers and making use of the various possible singlet state resonances in absorption and emission. So far (cf. fig. 1) most experiments, using the dyes rhodamine $6 \mathrm{G}$, rhodamine $B$, and sulforhodamine $B$, have been performed with the fundamental $\lambda_{0}$ off resonance and the surface-enhanced harmonic emission at resonance with one of the higher-lying singlet states. It is the purpose of this contribution to demonstrate for the first time that simultaneous resonance of the fundamental $\lambda_{0}$ with a $S_{0}-$ $S_{1}$ transition and the harmonic with a $S_{0}-S_{2}$ transition leads to another strong SH-enhancement. This

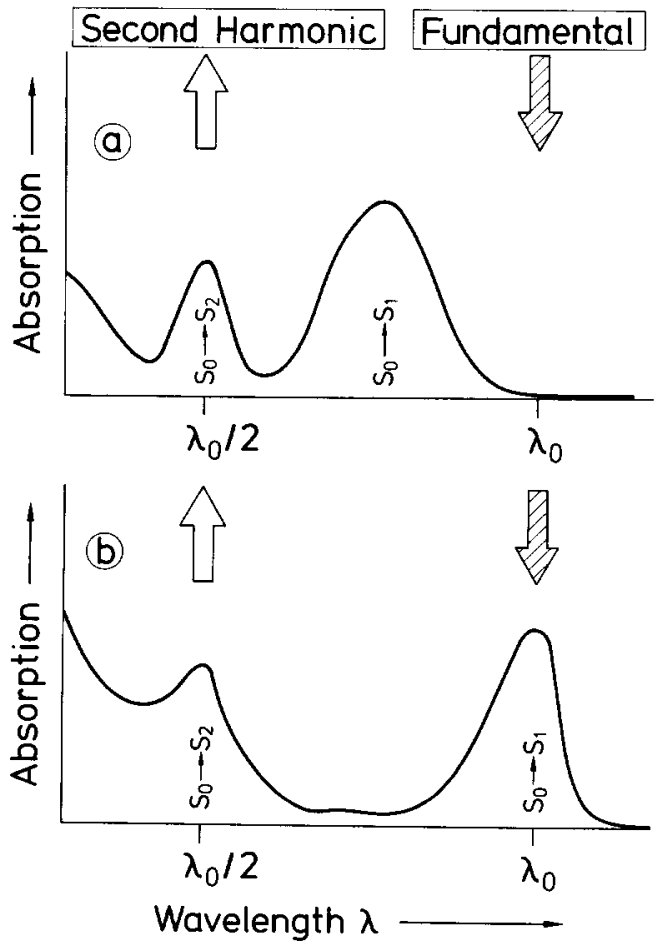

Fig. 1. Comparison of absorption spectra of adsorbed dyes used for SH-generation: (a) Harmonic emission is at resonance with the $S_{0}-S_{2}$ transition; (b) harmonic emission is at resonance with $S_{0}-S_{2}$ and the fundamental with $S_{0}-S_{1}$ groundstate $a b-$ sorption. 
will be shown with adsorbed monolayers of nileblue A, however similar results have been obtained under double-resonant operation of cresyl-violet.

Similar to the procedure outlined in refs. $[2,11,12]$ we consider as a model for SH generation by a layer of an adsorbate a thin slab of a nonlinear medium. Frequency conversion will be described in terms of a third rank tensor $\chi^{(2)}$. The resonant part of this second order susceptibility for the process of sum frequency generation is given by the expression [13]:

$$
\begin{aligned}
\chi_{i j k}^{(2)} & =\sum_{\mathrm{c}} \frac{M_{i}^{\mathrm{ca}}}{\omega_{\mathrm{ac}}+\omega_{1}+\omega_{2}+\mathrm{i} \Gamma_{\mathrm{ac}}} \\
& \times \sum_{\mathrm{b}}\left(\frac{M_{j}^{\mathrm{ab}} M_{k}^{\mathrm{bc}}}{\omega_{\mathrm{ab}}+\omega_{1}+\mathrm{i} \Gamma_{\mathrm{ab}}}+\frac{M_{k}^{\mathrm{ab}} M_{j}^{\mathrm{bc}}}{\omega_{\mathrm{ab}}+\omega_{2}+\mathrm{i} \Gamma_{\mathrm{ab}}}\right),
\end{aligned}
$$

where $\omega_{1}$ and $\omega_{2}$ are the frequencies of the ingoing light beams, $\omega_{\mathrm{ab}}=\left(E_{\mathrm{a}}-E_{\mathrm{b}}\right) / \hbar$ are molecular transition energies and $\Gamma_{\mathrm{ab}}$ the corresponding dephasing rates $T_{2}^{-1}(\mathrm{ab})$. The transition dipole moments are defined as:

$M_{i}^{\mathrm{ca}}=\left\langle\mathrm{c}\left|e R_{i}\right| \mathrm{a}\right\rangle$

and the indices $i, j$, and $k$ refer to the cartesian components of the polarization vectors:

$P_{i}=\sum_{j k} \chi_{i j k}^{(2)} E_{j}\left(\omega_{1}\right) E_{k}\left(\omega_{2}\right)$

The energy level diagram relevant for $\chi_{i j k}^{(2)}$ is depicted in fig. 2. The two terms in the curly brackets of eq.

(1) arise since we considered both time orderings for

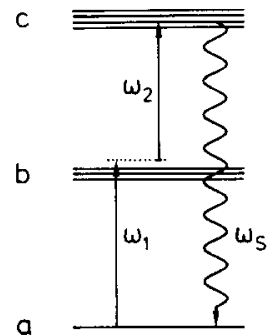

(a)

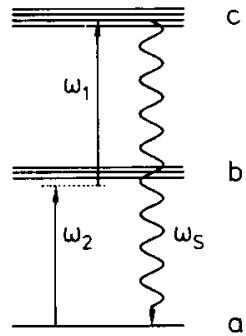

(b)
Fig. 2. Level diagrams showing both time orderings considered in deriving the resonant part of $x^{(2)}$ for sum frequency interaction and second harmonic generation. the interaction as indicated in fig. 2. This is necessary when the level spacings $a-b$ and $b-c$ are comparable to both frequencies $\omega_{1}$ and $\omega_{2}$. The summation over levels $b$ and $c$ in eq. (1) can be limited to the resonant states $S_{1}$ and $S_{2}$ in the case that well separated nondegenerate levels are present, i.e. the level spacings are much larger than the widths, and only the appropriate three level system is retained.

The special case of second harmonic generation is obtained by setting $\omega_{1}=\omega_{2}$ when both beams can be distinguished and only the signal generated by both beams together is detected. A situation of this kind is employed in background free autocorrelation experiments. When only one beam generates second harmonic, an additional factor $1 / 2$ arises:

$$
\begin{gathered}
\chi_{i j k}^{(2)}(2 \omega)=\frac{1}{2} \sum_{\mathrm{c}} \frac{M_{i}^{\mathrm{ca}}}{\omega_{\mathrm{ac}}+2 \omega+\mathrm{i} \Gamma_{\mathrm{ac}}} \\
\quad \times \sum_{\mathrm{b}} \frac{M_{j}^{\mathrm{ab}} M_{k}^{\mathrm{bc}}+M_{k}^{\mathrm{ab}} M_{j}^{\mathrm{bc}}}{\omega_{\mathrm{ab}}+\omega+\mathrm{i} \Gamma_{\mathrm{ab}}}
\end{gathered}
$$

since the intensity product $I_{1} I_{2}$ yields $(2 I)^{2}$ in this case.

With no real intermediate state $b$ being present the width $\Gamma_{\mathrm{ab}}$ can be neglected. The term in curly brackets then is the two-photon cross section of level $c$ and will be slowly varying with $\omega$. As a result, the resonances follow the absorption spectrum at $2 \omega$ :

$\left|\frac{M_{i}^{\mathrm{ca}}}{\omega_{\mathrm{ac}}+2 \omega+\mathrm{i} \Gamma_{\mathrm{ac}}}\right|^{2}=\operatorname{Im}\left\{\chi_{i i}^{(1)}(2 \omega)\right\} / \Gamma_{\mathrm{ac}}$.

In the double resonance case, however, the spectrum will be the convolution of both the $a-b$ and the $a-c$ absorption spectrum.

\section{Experimental}

The optical schematic of the experimental setup is shown in fig. 3. All experiments have been performed by pumping a narrowband, tunable dye laser (Lambda Physik FL 2000 [14]) with an excimer laser, operating at the $308 \mathrm{~nm}$ transition of $\mathrm{XeCl}$. Several dyes have been used (coumarine 153, rhodamine 6G, sulforhodamine $B$, and $D C M$ ), to cover the spectral range from 


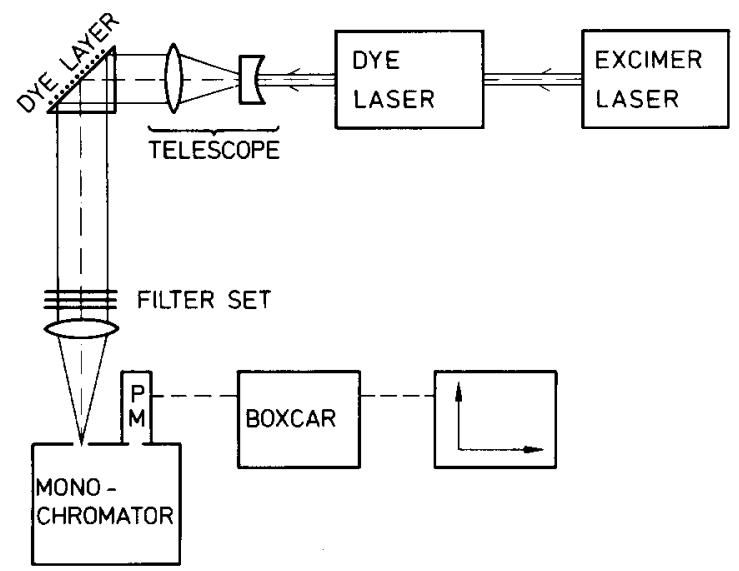

Fig. 3. Experimental setup.

$535 \mathrm{~nm}$ to $700 \mathrm{~nm}$. The pulse energy was kept constant to $1 \mathrm{~mJ}$ in any case and typical pulse duration were 10 ns. An adjustable telescope allowed irradiation of various spot sizes of the dye monolayer covered hypotenuse of a high quality quartz prism. For coverages with dyes with no groundstate absorption, e.g. rhodamine $6 \mathrm{G}$, the dye laser radiation was focused to $1.3 \times 10^{-3} \mathrm{~cm}^{2}$, whereas irradiation of nile-blue A required an enlarged spot size of $15.4 \times 10^{-3} \mathrm{~cm}^{2}$ to prevent laser induced damage. The UV-signal was detected by a monochromator-multiplier combination (3/4 m Czerny-Turner type Spex spectrometer and Valvo XP-2020 multiplier tube), shielded with a set of interference filters for rejection of the fundamental wavelength. The signal was finally recorded with a chart-recorder connected to a PAR boxcar averager. Highest signals were obtained by SHexcitation with s-polarized fundamental radiation and p-polarized UV-detection (details cf. [12]). Under these observation conditions the SH-signal of the quartz background was rejected by at least three orders of magnitude.

\section{Results and discussion}

The experimental results obtained with monolayer coverages of nile-blue A as SH-source are shown in figs. 4 and 5. Fig. 4 shows the spectral enhancement (dashed line) of the SH radiation together with an absorption spectrum of nile-blue A, dissolved in propanol (solid line). The peak of the spectral enhancement is centered at $300 \mathrm{~nm}$ and corresponds approximately to a 100 . fold increase in SH-signal strength, if the UV-signal obtained upon irradiation at $750 \mathrm{~nm}$ is taken for comparison. In the latter case no enhancement due to $S_{0}-$ $S_{1}$ absorption is effective, however a coincidence of the harmonic frequency with a $S_{0}-S_{2}$ transition is still present. In contrast to previous studies using $\mathrm{S}_{0}$ $\mathrm{S}_{2}$ enhancement (e.g. rhodamine $6 \mathrm{G}$ or sulforhodamine B) the spectral shape of the SH-enhancement does not resemble any particular feature of the nile-blue $\mathrm{A}$ ab-

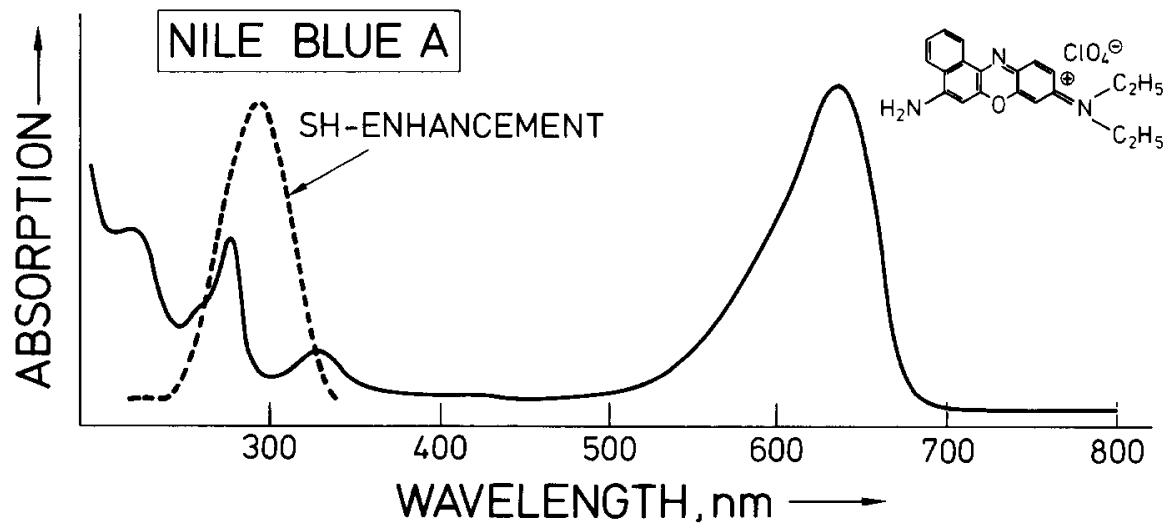

Fig. 4. Absorption spectrum of nile-blue A (chemical structure at upper right) together with observed SH-enhancement centered at $300 \mathrm{~nm}$. 


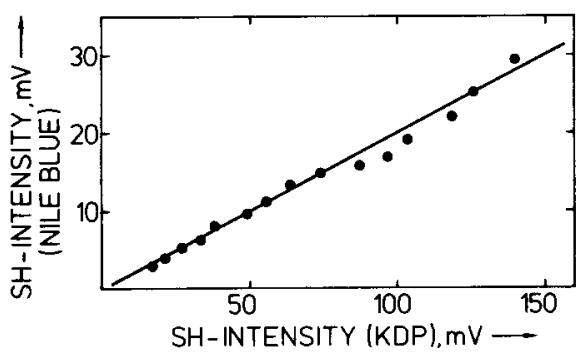

Fig. 5. Second harmonic intensity of nile-blue $A$ versus second harmonic intensity of a KDP crystal used for output normalization.

sorption spectrum $[1,10]$. In fact, the observed increase of the SH-signal is a spectral superposition of both, the wavelength-dependent enhancement due to absorption of the fundamental and the resonant emission of the harmonic as discussed in the introductory section. Fig. 5 depicts the linear dependence of the nile-blue A SH-signal versus the SH-signal of a KDP crystal of $1 \mathrm{~mm}$ thickness, operated under phasematched conditions. The KDP crystal was inserted between quartz prism and filter set and great care was taken to operate the frequency doublet under identical conditions as to beam divergence and effective cross section. In particular the quadratic power dependence of KDP UV-output on the pump laser input power was checked carefully. In our experiments normalization against the UV-signal of a KDP crystal was more reliable than the usual procedure of normalization against the peak of a Maker fringe pattern [15]. From fig. 5 it is immediately evident that a nile-blue A monolayer produces in its peak of UV-mission at $300 \mathrm{~nm}$ approximately $20 \%$ of the UV-radiation of a $1 \mathrm{~mm}$ KDP crystal operated under identical conditions.

Finally it is worthwhile to compare the conversion efficiency of a nile-blue A coverage with a single resonant coverage of rhodamine $6 \mathrm{G}$ with its peak of SHemission at $350 \mathrm{~nm}$ [2]. In order to eliminate spectral detector sensitivity and differences in pump intensity, all data are normalized against the respective KDP signals. This procedure yields an intensity ratio
$I_{\mathrm{UV}}$ (nile-blue A) $: I_{\mathrm{UV}}($ rhodamine $6 \mathrm{G})=140: 1$.

Considering the different spot sizes $\left(15.4 \times 10^{-3} \mathrm{~cm}^{2}\right.$ : $1.3 \times 10^{-3} \mathrm{~cm}^{2}=11.85$ ) and the resulting decrease in pump intensity for nile blue $A$, this results in an efficiency ratio per molecule $\eta_{U V}$ (nile-blue A) : $\eta_{\mathrm{UV}}$ (rhodamine 6G) $=1659: 1$, assuming equal coverage densities. Application of this rather high conversion efficiency due to double resonant spectral enhancement is only limited by severe laser induced damage problems, even under excitation in the above described total internal reflection.

\section{References}

[1] C.K. Chen, A.R.B. de Castro and Y.R. Shen, Phys. Rev. Lett. 46 (1981) 145.

[2] C.K. Chen, T.F. Heinz, D. Ricard and Y.R. Shen, Phys. Rev. Lett. 46 (1981) 1010.

[3] T.F. Heinz, C.K. Chen, D. Ricard and Y.R. Shen, Phys. Rev. Lett. 48 (1982) 478.

[4] T.F. Heinz, H.W.K. Tom, X.D. Zhu and Y.R. Shen, paper MCC2, Technical Digest XIII IQEC Anaheim, CA, 1984.

[5] H.W.K. Tom, C.M. Mate, X.D. Zhu, J.E. Crowell, T.F. Heinz, G.A. Somorjai and Y.R. Shen, paper MHH2, Technical Digest XIII IQEC Anaheim, CA, 1984.

[6] T.F. Heinz, H.W.K. Tom and Y.R. Shen, Phys. Rev. A28 (1983) 1883.

[7] H.W.K. Tom, T.F. Heinz, P. Ye and Y.R. Shen, in: Laser Spectroscopy, VI, eds. H.P. Weber and W. Lüthy, Proc. SICOLS, Interlaken, Switzerland, 1983 (Springer Verlag Berlin, Heidelberg, New York, Tokyo, 1983) p. 289.

[8] N. Bloembergen, Nonlinear optics (Benjamin, New York, 1977).

[9] G.A. Reider, A.J. Schmidt and G. Marowsky, Optics Comm. 47 (1983) 223.

[10] G. Marowsky, A. Gierulski, G.A. Reider and A.J. Schmidt, Appl. Phys. B34 (1984) 69.

[11] N. Bloembergen and P.S. Pershan, Phys. Rev. 128 (1962) 606.

[12] B. Dick, A. Gierulski and G. Marowsky, paper in preparation.

[13] B. Dick and R.M. Hochstrasser, J. Chem. Phys. 78 (1983) 3398.

[14] The dye laser FL 2000 was kindly left to our disposal by Dr. R. Vehrenkamp, Lambda Physik, Göttingen.

[15] P.D. Maker, R.W. Terhune, M. Nisenoff and C.M. Savage, Phys. Rev. Lett. 8 (1962) 21. 